\title{
Silicon Drift-Chamber Studies for Possible Lise at RHIC
}

\section{DISCLAIMER}

\begin{abstract}
This report was prepared as an account of work sponsored by an agency of the United States Government. Neither the United States Government nor any agency thereof, nor any of their employees, makes any warranty, express or implied, or assumes any legal liability or responsibility for the accuracy, completeness, or usefulness of any information, apparatus, product, or process disclosed, or represents that its use would not infringe privately owned rights. Reference herein to any specific commercial product, process, or service by trade name, trademark, manufacturer, or otherwise does not necessarily constitute or imply its endorsement, recommendation, or favoring by the United States Government or any agency thereof. The views and opinions of authors expressed herein do not necessarily state or reflect those of the United States Government or any agency therenf.
\end{abstract}

T. J. Humanic

University of Pittsburgh

Department of Physics and Astronomy

31 October 1990 


\section{TABLE OF CONTENTS}

1. Introduction

2. Semiconductor Drift Detectors

3. Progress Report

4. Test Program

5. Personnel and Schedule

6. Budget

7. Vitae of the Principal investigator 


\section{LIST OF FIGURES}

Figure 2-1: Perspective view of a silicon drift detector. Electrons created by an 3 ionizing particle are transported parallel to the detector suriace. The anode is divided into short segments to measure the coordinate perpendicular to the drift direction

Figure 3-1: Digitizer readout of four adjacent anodes with laser pulses incident on 6 the silicon "drift detector. Note that pulses are negative.

Figure 3-2: Drift time versus position for two different field settings. $170 \mathrm{~V} / \mathrm{cm}^{--} \quad 7$ star. and $230 \mathrm{~V} / \mathrm{cm}--\mathrm{cross}$

Figure 3-3: Simulation of the path deviation of electrons in a $0.8 \mathrm{~T}$ field for a particle hit at $5.2 \mathrm{~mm}$ from the anodes. A deflection of about 2 anodes is seen.

Figure 3-4: $\operatorname{Tan}(\theta)$ vs. B-field measured on our prototype drift chamber. The drift field was $531 \mathrm{~V} / \mathrm{cm}$.

Figure 3-5: Hall mobility versus $B-f i e l d$ and drift field. circle--185 V/cm. 11 star-- $295 \mathrm{~V} / \mathrm{cm}$. cross $--531 \mathrm{~V} / \mathrm{cm}$. Note that B-field is perpendicular to the surface of the drift detector

Figure 3-6: Election mobility versus $B-f i e l d$ and drift field. circle--185 V/cm. 12 star--295 V/cm. cross--531 V/cm

Figure 3-7: Hall factor versus B-field for a drift field of $295 \mathrm{~V} / \mathrm{cm}$. The crosses represent laser pulses that simulate a minimum ionizing particle, and the stars represent three times minimum ionizing measurements.

Figure 3-8: Reconstruction Accuracy for 1 and 2 hits randomly placed within an 14 Anode

Figure 3-9: Seperation between the 2 randomly placed hits when the reconstruction error was small. and when it was large

Figure 3-10: The efficiency for identifying and fitting a hit signal in the presence of other pileup hits is shown. Here the method of identifying a hit uses a center of gravity calculation about a peak. and subtraction according to the know amplitude for a minimum ionizing hit.

Figure 3-11: The sigma on the accuracy of identifying the drift time when a hit signal is fitted in the presence of other pileup hits is shown. Here the method of identifying a hit uses a center of gravity calculation about a peak, and subtraction according to the know amplitude for a minimum ionizing hit. 


\begin{abstract}
Summary
It is proposed to continue the prngram now underway at the University of Pittsburgh to study the feasibility of using silicon drift-chambers as particle tracking devices at RHIC. We are currently lesting a UA6-type detector obtained from BNL. and plan to also study a new device that will become available this lear: a cylindrical geometry detector designed for NA45 (CERN). In addition. we propose to fabricate and study a detector to be used in vertex determination for the RHIC OASIS experiment. The two-year budget for this proposal is $\$ 246.962$.
\end{abstract}




\section{Introduction}

The silicon drift-chamber [1] represents a new technology which offers great promise as a tracking device in a high-multiplicity environment such as found at RHIC. Since March of 1989. We at the University of Pittsburgh have embarked on a study of such a device. a UA6lype detector prototype obtained from P." Rehak of BNL. We have obtained some preliminary results with this detector that look promising and would like to continue our lests of this detector. In addition, we would like to test a new device of cylindrical geometry that is being fabricated for the CERN NA45 experiment as a tracking element in an electron spectrometer. We will also be involved in fabrication and testing of a detector to be used for a vertex determination for the RHIC OASIS experimemt in which the principle investigator is a member. In these tests. we will continue our collaboration with P. Rehak at BNL. one of the inventors of the silicon drift-chamber. with whom we also plan to collaborate with on SSCrelated applications. Because of the similarities between the RHIC and SSC experimental environments. we think the knowledge gained in our tests will benefit both projects.

The remainder of this proposal is organized into five sections: a brief review of the principles of silicon drift chambers. a progress report on the lesting that we have done so far. an outline of the test program that would be carried out in the proposal period. a discussion of the personnel involved and a schedule, and a break-down of the budget.

\section{Semiconductor Drift Detectors}

Silicon drift detectors are a relatively new kind of semiconductor detector able to provide lery precise position and ionization measurements with a very modest amount of electronics. A perspective view of the drift detector is shown in Figure 2-1. In principle. the electric field of the drift detector forces electrons liberated by an ionizing particle 10 drift parallel to the large semiconductor surface to the anode. The transit tıme of electrons inside the detector measures the distance of an incident paricle from the anode. 


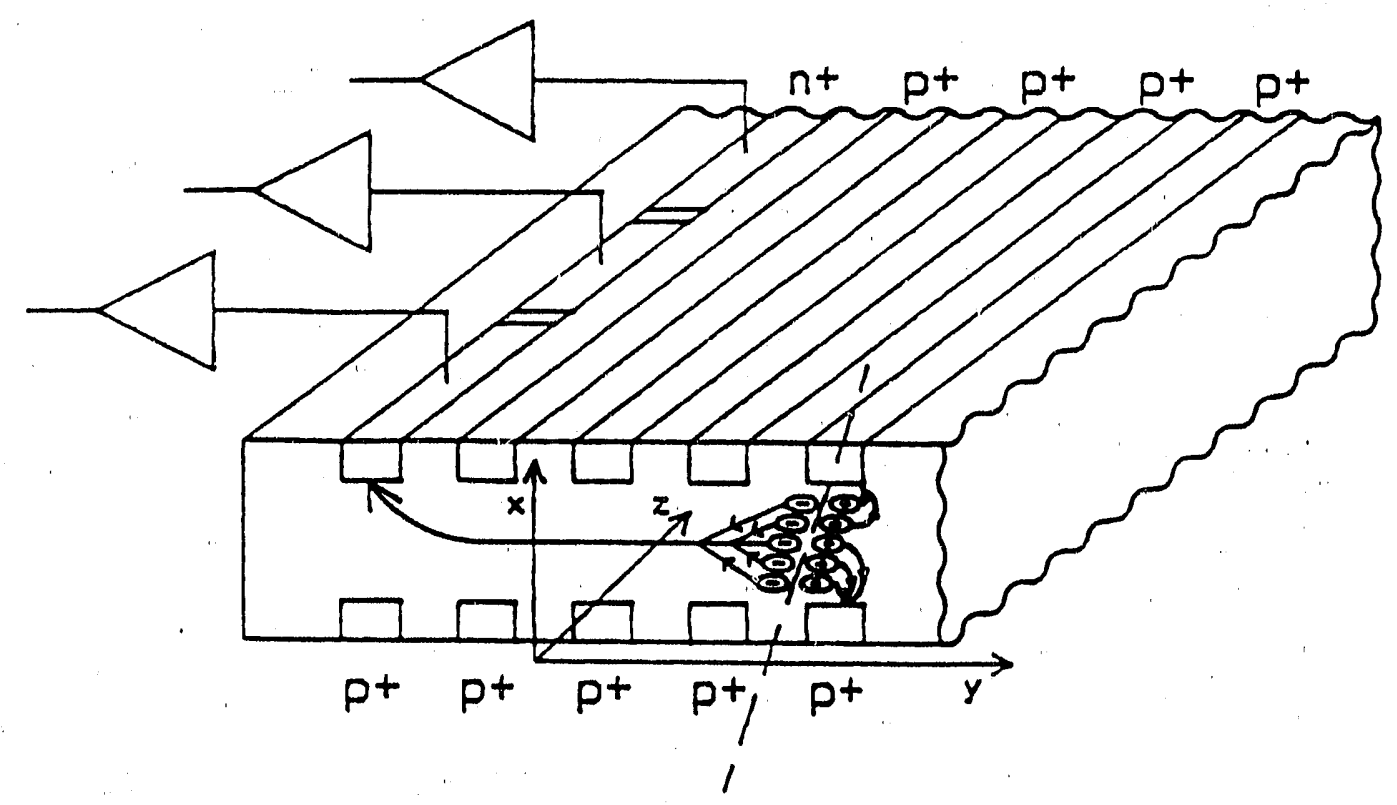

Figure 2-1: Perspective view of a silicon drift detector. Electrons created by an ionizing particle are transported parallel to the detector surface. The anode is divided into short segments to measure the coordinate perpendicular to the drift direction

As a direct consequence of this electron transport method the anode capacitance is much lower than the anode capacitance of the classical semiconductor detector for the same dimensions. The amplifier noise can be made much smaller which is the main reason behind the excellent position resolution of silicon detectors.

The measured position resolution in a test beam was 10 micro- meters on the drift distance of 4000 micrometers. These results [2] were achieved with external amplifiers which were not matched to the very low anode capacitance.

As an example, the drift detector to be used in the UA6 experiment at CERN SPPS has an area of $4 \mathrm{~cm} \times 4 \mathrm{~cm}$. maximum drift time of 1.5 microseconds, total number of anodes 332 and an unambiguous $x-y$ position resolution of 10 micrometers in both directions. 
It should be stressed that the maximum drift time is not the dead time of the silicon drift detector. Due to the fact that all electrons are continuously drained at the anode the silicon drift detectors have no dead time. Electrons drifting loward the anode have their charges screened by the electrode structure of the silicon drift detector and do not interfere with the other signals or with the signal processing at the anode. The signals are stored within the detector for the duration of their drift.

Figure 2-1 shows the collection anode divided into individual pad-anodes. The position of the responding anode(s) measures the coordinate perpendicular to the drift direction. Due to the transverse diffusion. the electrons produced by the ionization arrive as a gaussian shaped charge cloud in the anode region. Charge will be usually collected on more than one anode. The charge division method yields the position of the crossing particle in the second coordinate with a precision down to about $4 \%$ of the anode-pad pitch. This multi-anode silicon drift detector provides an unambiguous two dimensional position information. The postion information is similar to one which is to be expected from proposed silicon pixel devices. The multi-anode silicon drift detector gives better resolution with four orders of magnitude less read-out channels.

\section{Progress Report}

In March of 1989 we received RHIC R\&D funding to begin our study of silicon driftchambers. Since then our main accomplishments have been to establish a collaboration with P. Rehak of BNL. set up a testing lab at Pittsburgh, and carry out some preliminary tests on an actual device. As mentioned above, the device we are presently using is a developmental detector which served as a protolype for a CERN/Milano effort 10 develop a practial device for use in the LAb experiment. Although the prototype detector is smaller in area (1x1.6 sq.cm) compared with the UA6 detector $(3.2 \times 4$ sq. $\mathrm{cm})$ its performance characterists are 
considered. at this point. to be better than the UAb detector and thus better suited for our tests to see if such a device is useful for the RHIC environment. The detector. one of five such detectors in existance, was obtained from Rehak at BNL. After some initial dark current testing at BNL. we brought the detector to Pittsburgh to electrially bond it to a pc-board for the further lests (two other detectors were also bonded at Pittsburgh for BNL). We also borrowed some readout electronics from BNL consisting of preamplifiers and gaussian shapers. The rest of the equipment for the test lab was purchased with DOE and University money, and consists of:

- a $4 \times 6$ sq.foot optical table for mechanical stability and easy fixturing

- a pulsed Nd:Yag laser producing a fast (6ns width) pulse in the infrared $(1064 \mathrm{~nm})$ suitable to simulate the effects of a mininum ionizing particle in the detector

- a four-channel $200 \mathrm{Mhz}$ time digitizer to digitize the signals from four readout anodes of the detector in $5 n$ s bins to study their pulse shapes

- an IBM XT clone that is connected to the digitizers via an RS-232 link that is used for fulther pulse shape analysis

- optics to focus the laser beam to a several micron spot on the detector

- a comiputer-controlled $x-y$ stage to precisely position the detector under the laser beam

- miscellaneous electronic equipment such as a NIM-bin. power supplies, voltmeters. etc..

The infraced pulse from the laser is brought to the focussing optics via light fibers which help define the eventual size of the focussed spot on the detector and which. in groups of fibers. can be $\|$ sed to simulate multi-particle hits on the detector. In order to do multihit studies, it is desirable to read out as many detector anodes as possible. Recently, we have upgraded our readout electronics by purchasing new low-noise preamplifiers and faster gaussian shapers (sigma $=30 \mathrm{~ns})$ and added two more channels of digitizers.

In the remainder of this section. We will present preliminary results from the setup described above in four areas: 
- position versus time measurements

- two-track resolution measurements

- measurements in a magnetic field

- Monte Carlo simulations

\section{a. Position versus Time Measurements}

Figure 3-1 shows pulse shapes from their respective gaussian shapers (negative pulses) of four ajacent anodes from incident laser pulses. Three pulses are seen in each anode corresponding

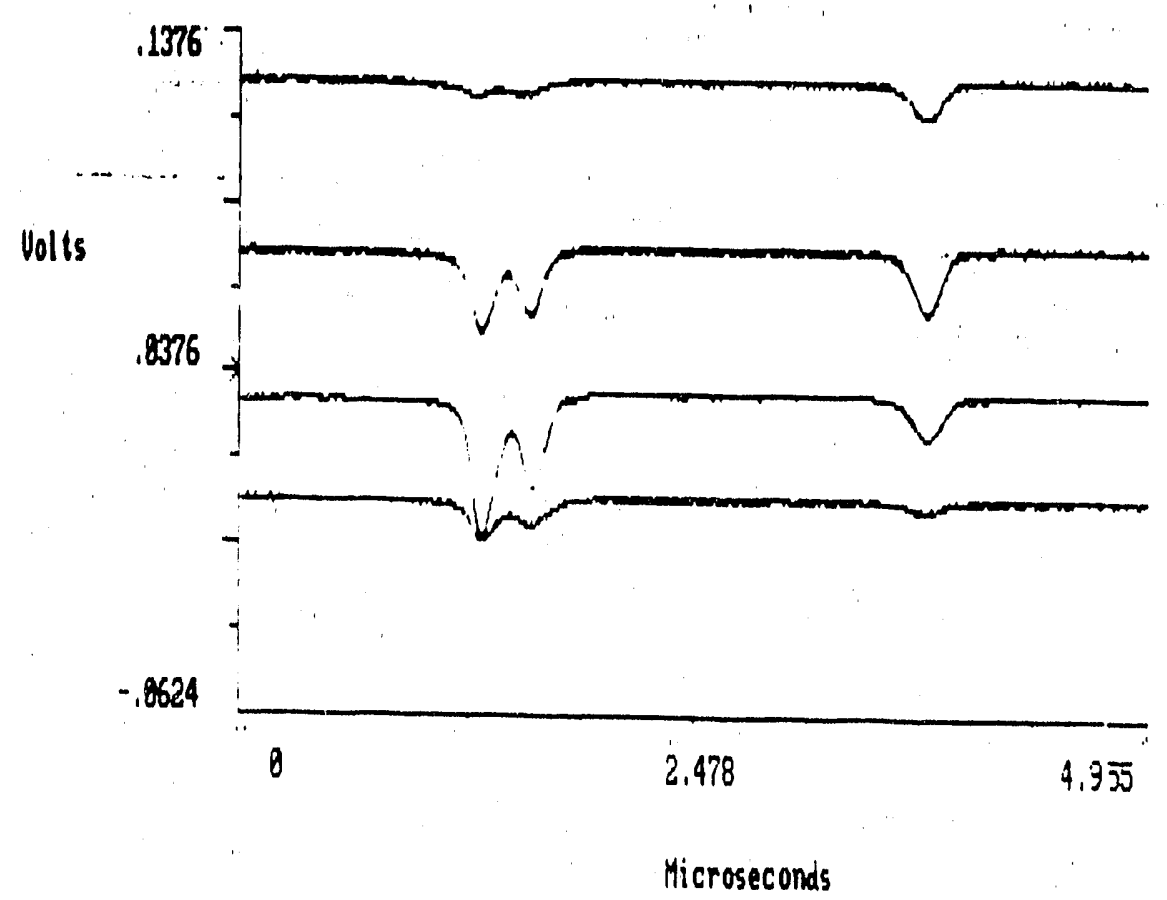

Figure 3-1: Digitizer readout of four adjacent anodes with laser pulses incident on the silicon drift detector. Note that pulses are negative.

to three optical fibers which guided the laser light to positions $4.71 \mathrm{~mm}$. $5.64 \mathrm{~mm}$, and $13.4 \mathrm{~mm}$ along the drift direction of the silicon drift detector. Although each light pulse was focused onto the detector to a 5 micron spot. charge is seen to leak into adjacent anodes (Anodes are 
250 microns apart.) showing the lateral size of the electron cloud for each pulse to be of order" > 250 microns. Note that the intensities of the laser pulses were adjusted to simulate as closely as possible the charge produced by minimum ionizing particles, i.e. about 25,000 electrons. A drift field of $300 \mathrm{~V} / \mathrm{cm}$ was used for the measurement, resulting in a drift velocity for the electrons of $3.57 \mathrm{~mm} /$ mictosecond. Using data like this, we produced the drift time versus laser beam position (along drift-field) plot shown in Figure 3-2. Plots are

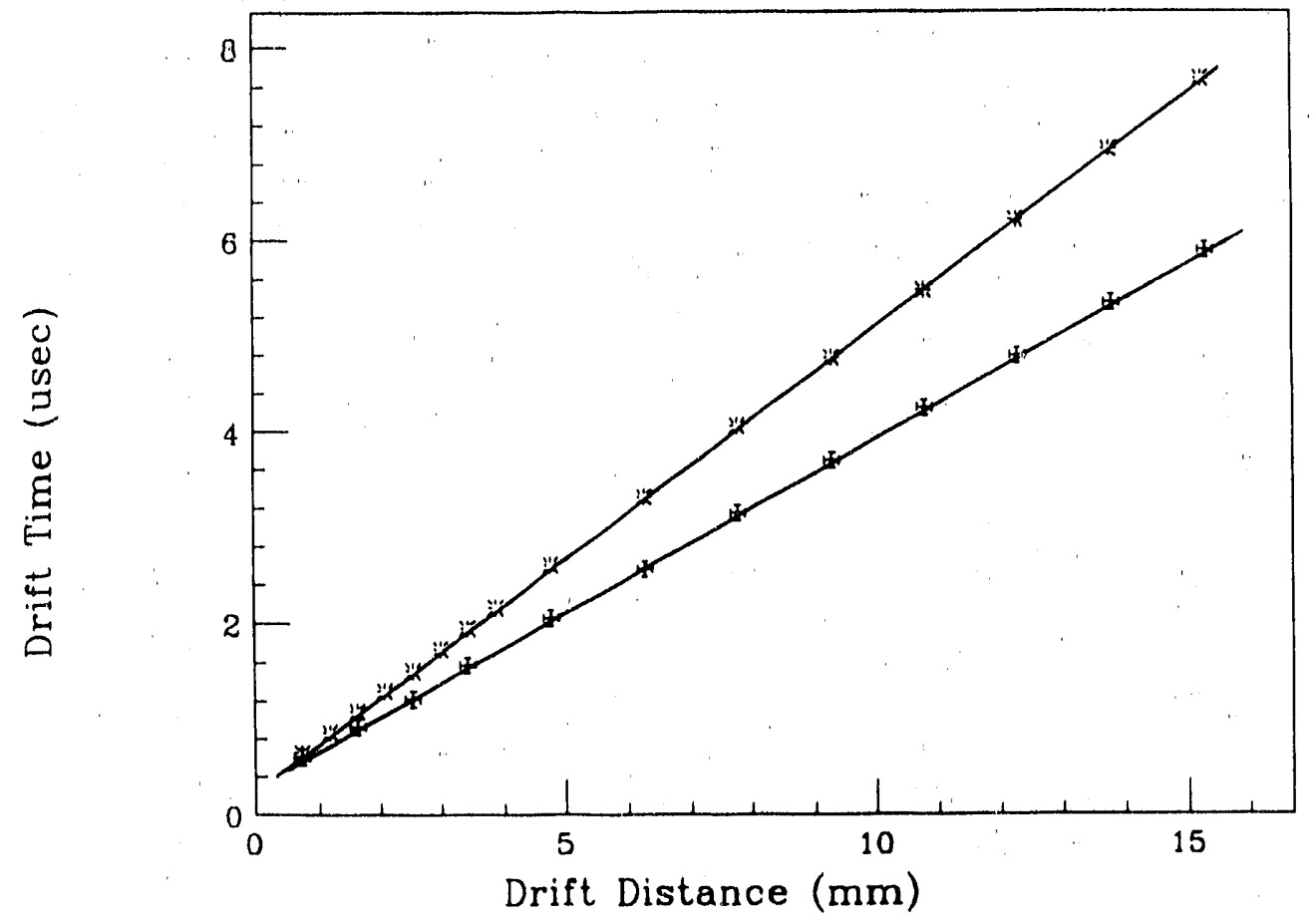

Figure 3-2: Drift time versus position for two different field settings, $170 \mathrm{~V} / \mathrm{cm}--\mathrm{star}$. and $230 \mathrm{~V} / \mathrm{cm}--$ cross

shown for two different drift fields. $170 \mathrm{~V} / \mathrm{cm}$ and $230 \mathrm{~V} / \mathrm{cm}$. The plots are seen to be quite linear throughout the measured ranges, resulting in a position error for a particular time on the order of 10 microns. We have also examined position readout in the anode direction using charge division between anodes and get a similar results for that coordinate. These results agree with previous measurements and are close to theoretical expections [2]. 


\section{b. Two-track reselution measurements}

As mentioned above. Figure 3-1 shows three laser pulses at various distances along the drift direction. Two of the pulses were set to be about 930 microns apart to study the two-track resolution of the detector. We used two gaussians to fit to these peaks to see what separation we measure from the drift" chamber and then compared it with the known separation. The result from our fit for the separation is 930 microns with a $1.5 \%$ fit error, in agreement with the value set. We have also made tests with separations as small as 500 microns and have sucessfully resolved these peaks with similar errors. These results are in agreement with the predictions of Gatti et al. [3]. We consider this work to be preliminary but encouraging and plan to carry out a more systematic two-track resolution study in the near future.

\section{c. Measurements in a Magnetic Field}

Because these detectors might find applications as particle trackers in the magnet fields of spectrometers (as in the OASIS experiment, see below) it is important to study the effects of magnetic fields on the operation of silicon drift chambers. Since no measurements of this kind existed. we decided to perform a series of measurements with our prototype detector in a 1 Tesla magnet that exists at the University of Pittsburgh. A range of magnetic fields were used from $1 \mathrm{~T}$ to $1 \mathrm{~T}$, the direction of the field always being perpendicular to the surface of the detector to see the maximum effect on the drifting electrons. Figure 3-3 shows a calculation of how the path of the electrons in a drift chamber is modified by a $0.8 \mathrm{~T}$ field for a laser pulse (or particle), incident at $5.2 \mathrm{~mm}$ from the anodes. The deflected path, deflected by an angle $\theta=6.4$ deg. from the no-field case. is a straight line that is offset by $0.58 \mathrm{~mm}$ at the readout anodes, i.e. about a two anode deflection. For this calculation a drift field of 531 $\mathrm{V} / \mathrm{cm}$ and electron mobility in silicon of 0.14 meter $/(\mathrm{V}-\mathrm{sec})$ were used. We have measured 


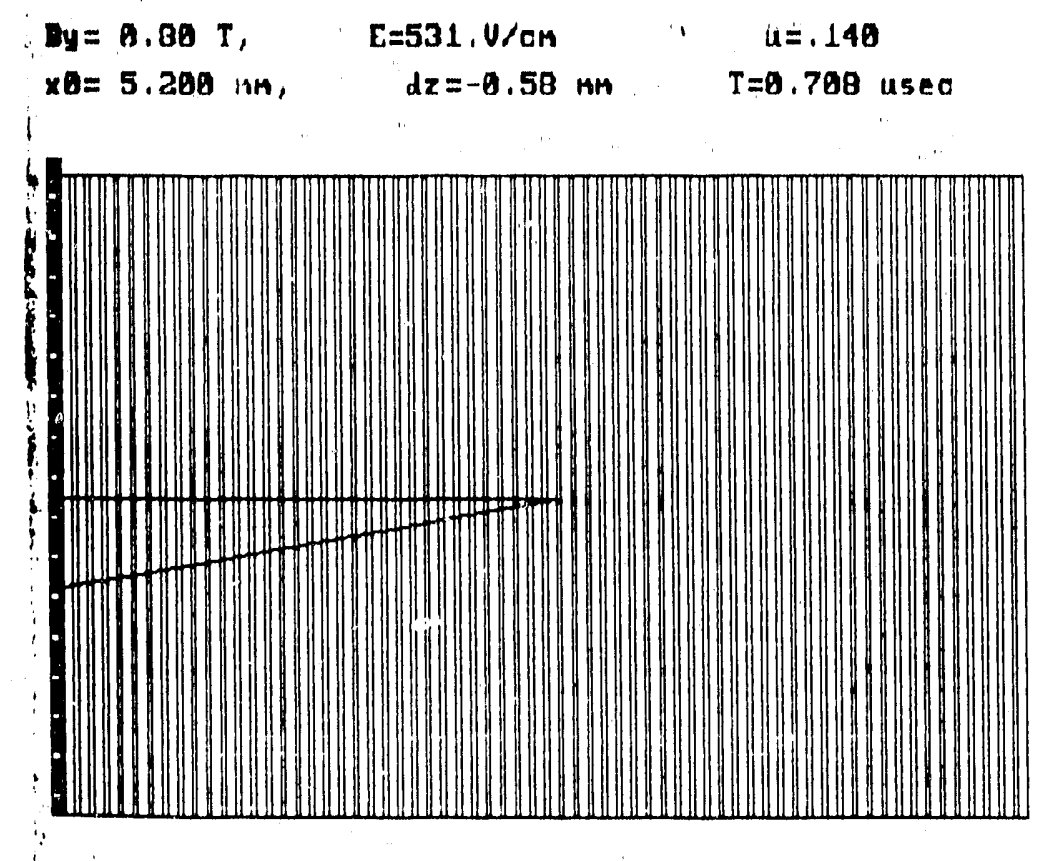

Figure 3-3: Simulation of the path deviation of electrons in a $0.8 \mathrm{~T}$ field for a particle hit at $5.2 \mathrm{~mm}$ from the anodes. A deflection of about 2 anodes is seen.

$\tan (\theta)$ versus B-field for a range of fields and get the results shown in Figure 3-4: The Hall mobility, $\mu_{H}$ is given by the ratio $\tan (\theta) / B$ and is expected to be close in value to the electron mobility. $\mu$. We used measurements like those shown in Figure 3-4 to obtain the Hall mobility versus $B$ for several different drift fields. The results are shown in Figure 3-5: In Figure 3-5. three different drift fields are shown. $185 \mathrm{~V} / \mathrm{cm} .295 \mathrm{~V} / \mathrm{cm}$. and $531 \mathrm{~V} / \mathrm{cm}$. Within the error bars. $\mu_{H}$ seems independent of the applied B-field, though it does depend on the drift field--the larger the drift field. the smaller $\mu_{H^{\prime}}$ The Hall mobility for electrons for high-resistivity silicon has been previously measured using the Hall effect at room temperature $(300 \mathrm{~K})$ to be 0.145 meter/(V-s) [4]. This is seen in Figure $3-5$ to be close to what we measure using the method described above. Our dependence on drift voltage is thought to be caused by heating of the drift chamber by the implanted resistive divider at the higher drift voltages. since it is found in the literature that $\mu_{1 i}$ has a strong temperature dependence [5]. 


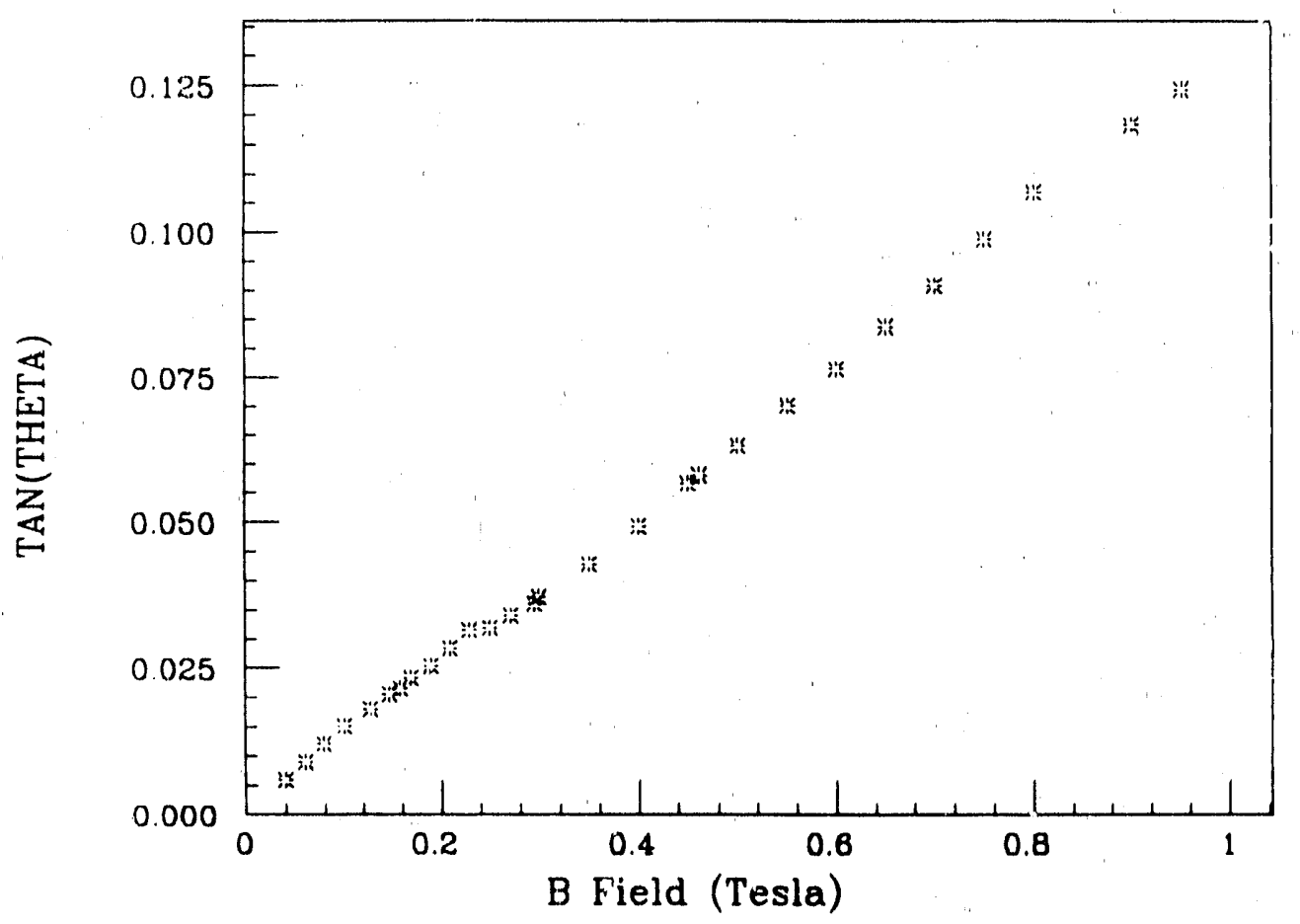

Figure 3-4: $\operatorname{Tan}(\theta)$ vs. B-field measured on our prototype drift chamber. The drift field was $531 \mathrm{~V} / \mathrm{cm}$.

In our next series of measurements. we plan to monitor the temperature of the silicon drift chamber to study this dependence. As a comparison to the Hall mobility, we have also measured the electron mobility, $\mu$, as a function of B-field and drift field, as shown in Figure 3-6: The electron mobility has been extracted from information like that obtained in Figure 3-2. i.e. from the drift velocity and applied drift field. The electron mobility is also seen to be independent of B-field but strongly dependent on drift field. A quantity which is of ten defined is the Hall factor which is the ratio $\mu_{H} / \mu$. This is plotted in Figure 3-7 versus Bfield for a drift field of $295 \mathrm{~V} / \mathrm{cm}$. The Hall factor is seen to be about 1.2 for this drift voltage. which is close to a theoretical prediction made by Long [5] at room temperature $(300 \mathrm{~K})$. Note that this ratio is not rery sensitive to the intensity of the laser pulse (ste figure caption). 


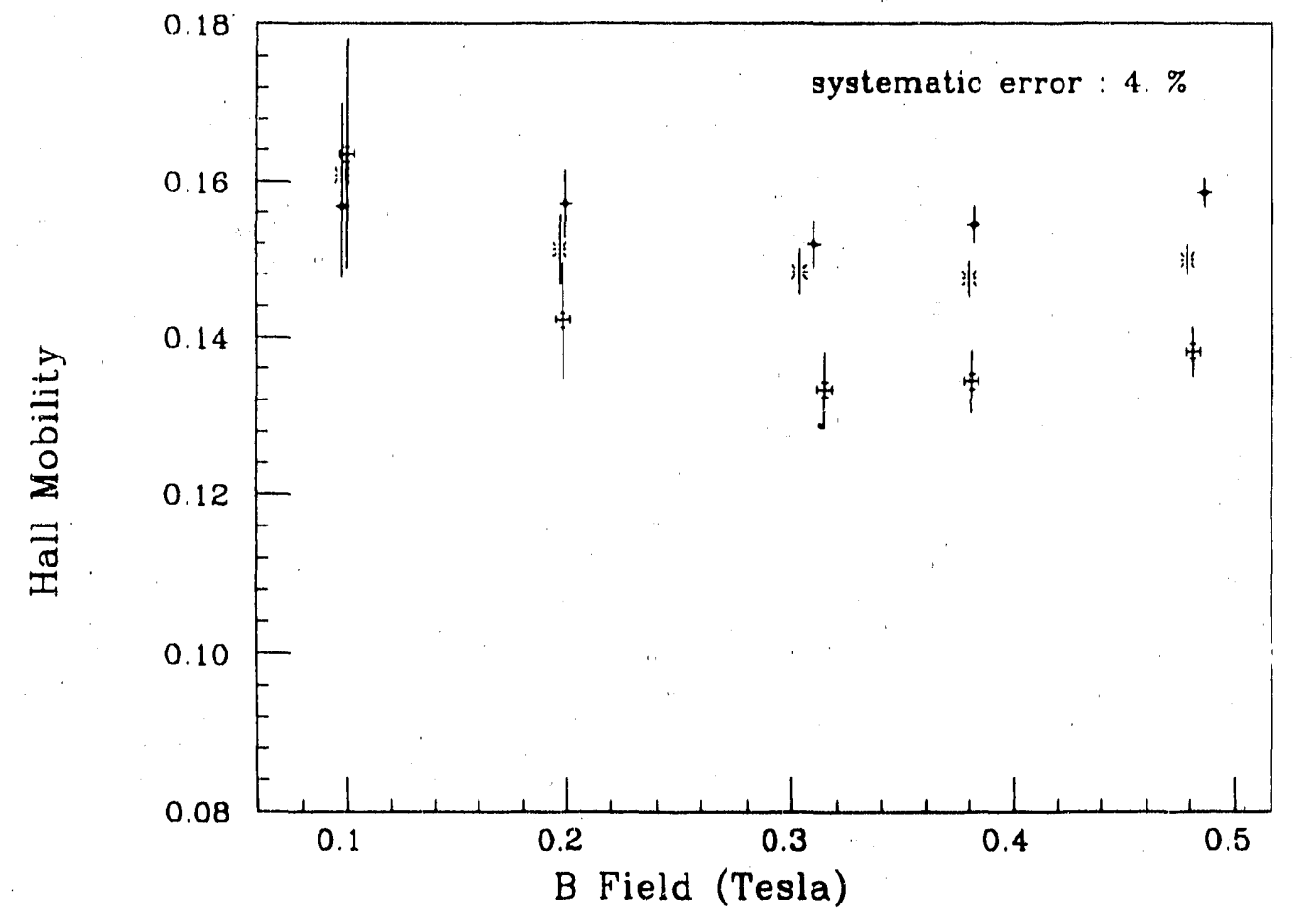

Figure 3-5: Hall mobility versus B-field and drift field. circle-- $185 \mathrm{~V} / \mathrm{cm}$. star--295 $\mathrm{V} / \mathrm{cm}$, cross $-531 \mathrm{~V} / \mathrm{cm}$. Note that $\mathrm{B}-\mathrm{f}$ ield is perpendicular to the surface of the drift detector

\section{d. Monte Carlo Simulations}

The Monte Carlo of the Silicon Drift Chamber was done within the GEANT framework. Two generators were used ISAJET and a simple few-particle generator used for cross-checking. The detector modelled is the 8 anode Generic detector sized to fit a 4 inch wafer.

The Monte Carlo consisted of two parts. The first was a generator and tracking program that found the hits in the detectors. made the digitizations and wrote the results to tape. The second was a reading and reconstruction program that read this "data" back. The original hit banks were also included on tape to compare to the reconstructed results. Two detectors were modelled simultaneously in any one tape writing job - one detector in any position required 


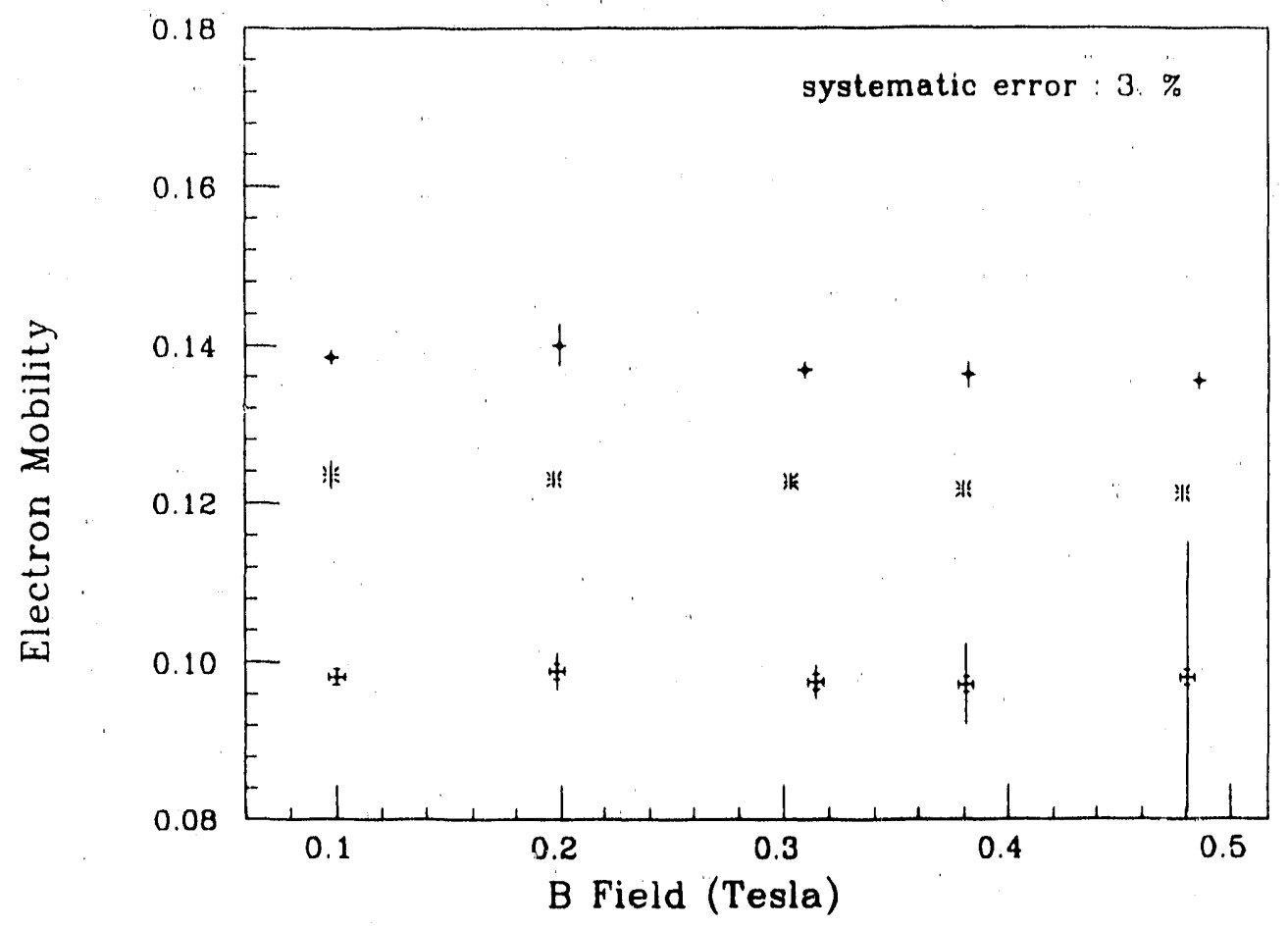

Figure 3-6: Electron mobility versus $B-f$ ield and drift field. circle--185 V/cm. star--295 $\mathrm{V} / \mathrm{cm}$. cross--531 V/cm

and another parallel detector immediately behind the first. The anode placement was identical in the parallel detector, but with the opposite drift direction to reconstruct the beam crossing time.

The detector performance was modelled using a parameterization of the drift of the charge left by the original ionizing particle. The width of the charge cloud as it drifts has been calculated and compared to measurements, as a function of total charge and total drift time. This time evolution of the charge cloud accounted for selt-repulsion and diffusion. The position of a 'hit' tracked to the detector was then 'drifted' in software towards the anode region. and a fake Flash ADC 'digitized' the charge cloud in 16 nanosecond steps. The 16 nanosecond steps were chosen somewhat arbitrarily. This is roughly the level of the digitization time anticipated at RHIC and the SSC. 


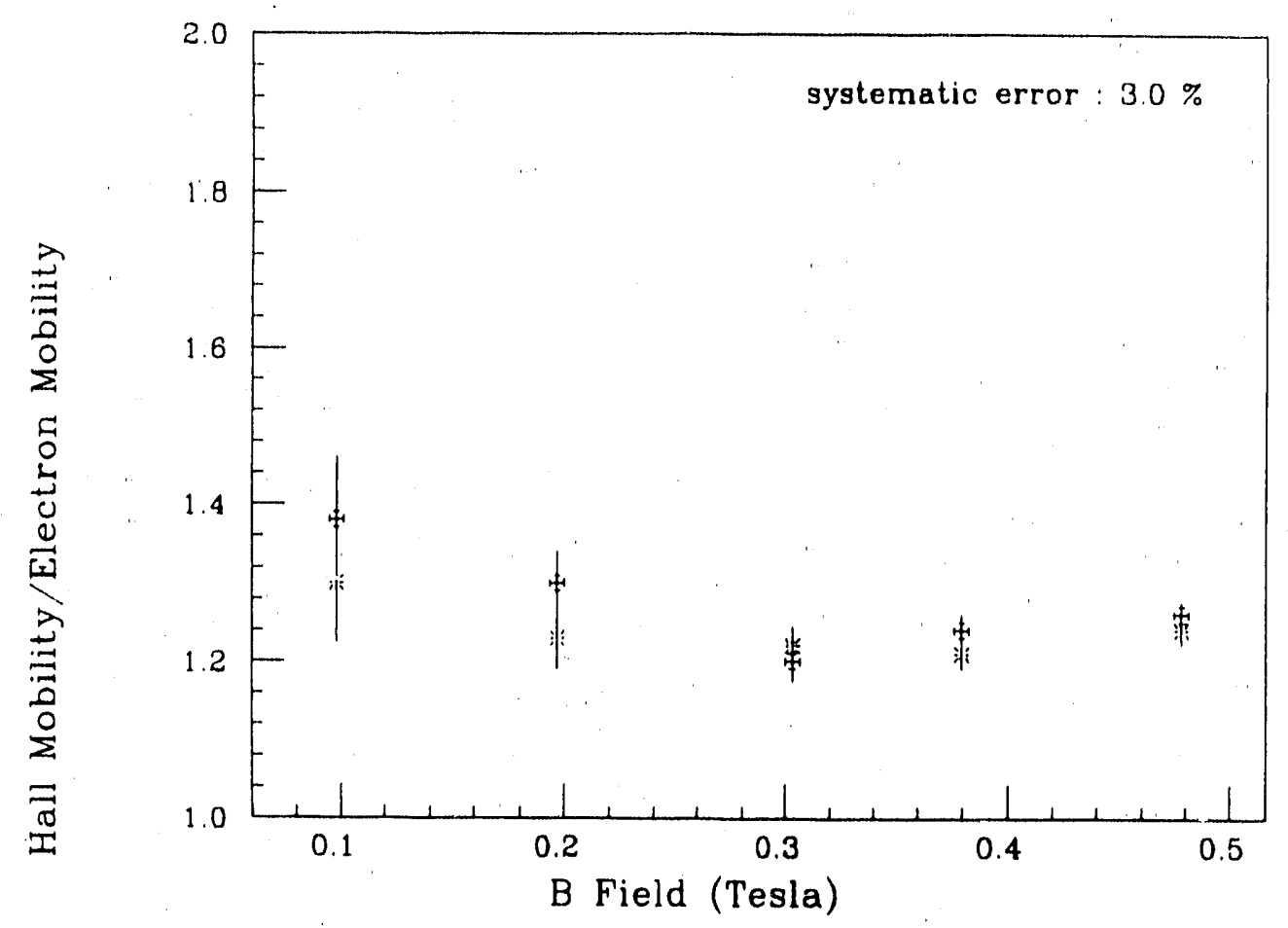

Figure 3-7: Hall factor versus B-field for a drift field of $295 \mathrm{~V} / \mathrm{cm}$. The crosses represent laser pulses that simulate a minimum ionizing particle, and the stars represent three times minimum ionizing measurements.

The drift electric field was chosen to be $600 \mathrm{~V} / \mathrm{cm}$ (for a drift velocity of the charge carriers of 7.98 microns/nsec). The software simulated a gaussian shaping amplifier with a shaping sigma of $20 \mathrm{nsec}$. Due to the diffusion and repulsion of the charge cloud. the typical width of the gaussian signals af ter the digitizing is nearer to 30 nsec.

The reconstruction program would sequentially scan each digitization stored on tape and search for the expected gaussian-shaped response of a hit. The center of the gaussian response was fit using both a simple center of gravity calculation and also a chi-squared minimization with the fitting program MINUIT. The result was compared to the original hit banks. This method was repeatedly applied to a given event. until all signals over a certain threshold were found and subtracted. 
The strategy of the Monte Carlo was to start with the generation of a single particle to compare the digitization for this single hit with that expected. The reconstruction efficiency and accuracy were found, to judge the method. Two particles were then simulated from the same bunch crossing to judge the interference in reconstructing each position and the bunch crossing time. Two particles but from different bunch crossings were then generated.

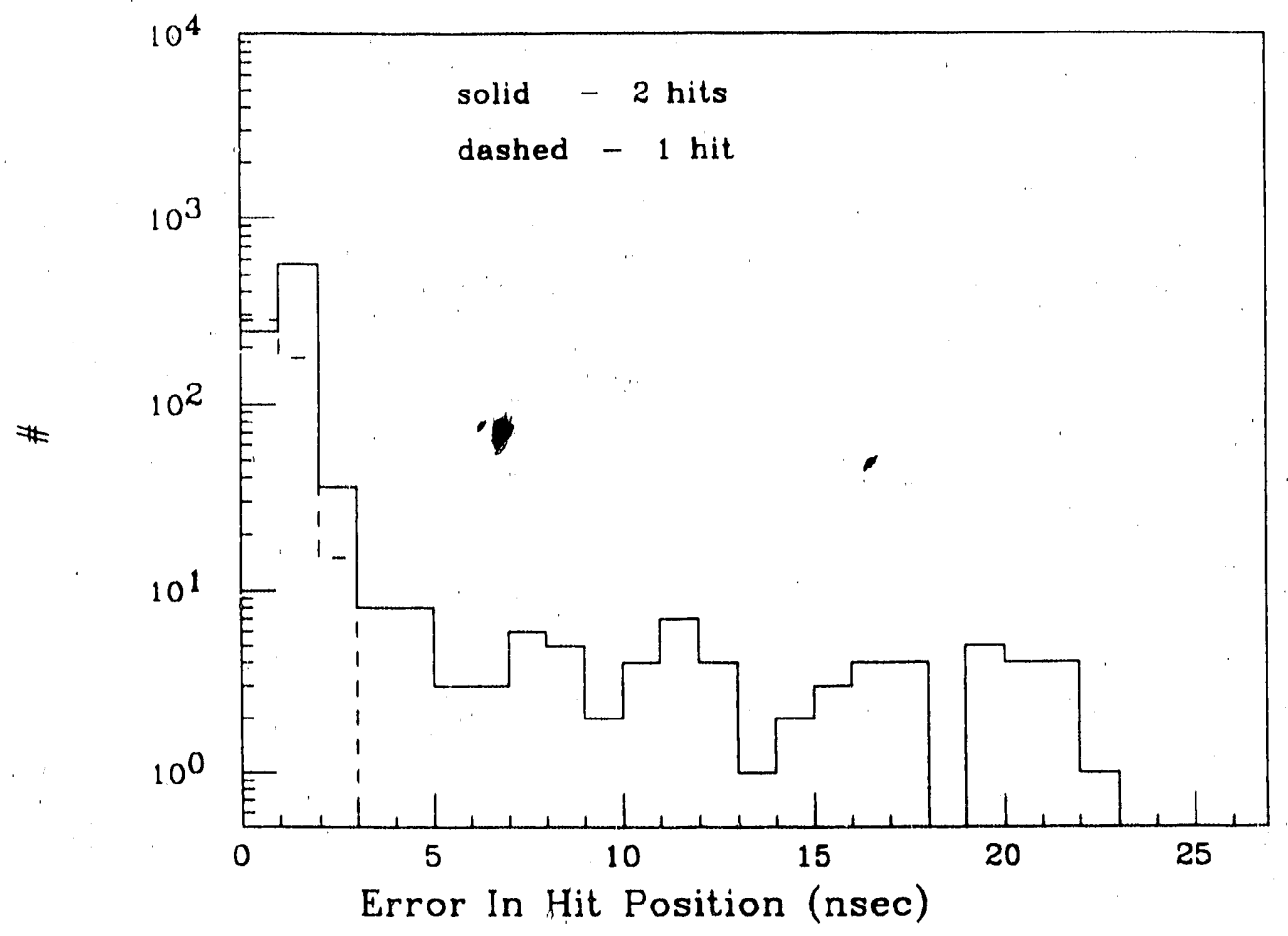

Figure 3-8: Reconstruction Accuracy for 1 and 2 hits randomly placed within an Anode

Figure 3-8 shows the error in the reconstruction of a hit. Note carefully that the vertical scale is logarithmic. Each hit has an expected drift time: the difference between this time and the reconstructed drift time is shown here in nanoseconds. $A$ bin of 1 nsec here corresponds to 8 microns. The 2-hit case shows considerable interference in the drift time reconstruction. Figure 3-9 shows the spatial seperation between the 2 hits. for 2 cases. The first case, dashed, is where the drift time reconstruction error in Figure 3-8 was less than 5 nsec for both hits. The solid line histogram shows the case where the drift time reconstruction is greater than 5 nsec. 


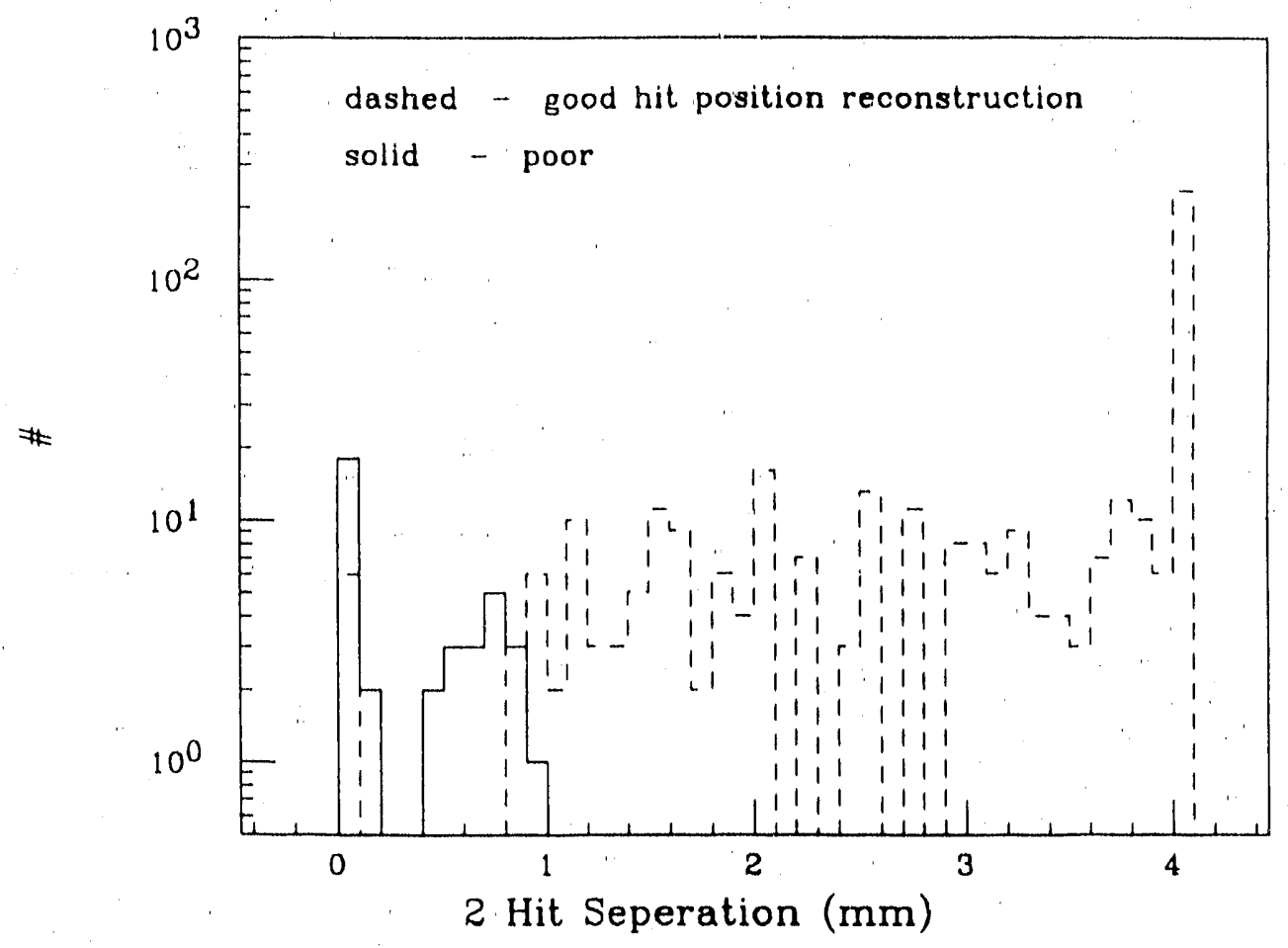

Figure 3-9: Seperation between the 2 randomly placed hits when the reconstruction error was small. and when it was large

The drift time reconstruction of the 2 hits becomes poor when their sejeration from each other is less than about $1 \mathrm{~mm}$. With an average sigma of 30 nsec. we can easily calculate that this corresponds to about 4 sigma seperation between the two gaussian signals. This perhaps is at the level that one may anticipate problems to begin in this simple method. The sum of the two signals each 2 sigma from their respective peaks becoming appreciable.

What has been demonstrated then is that with a simple method, a reasonably accurate reconstruction can be made of 1 and 2 particles in an anode. The failures even with this method are at the few percent level. What hasn't been shown is that in a real environment, pileup would not eventually kill all ability to identify these hits at all.

The ability to reconstruct many hits per anode was also tested with our simulation. the 


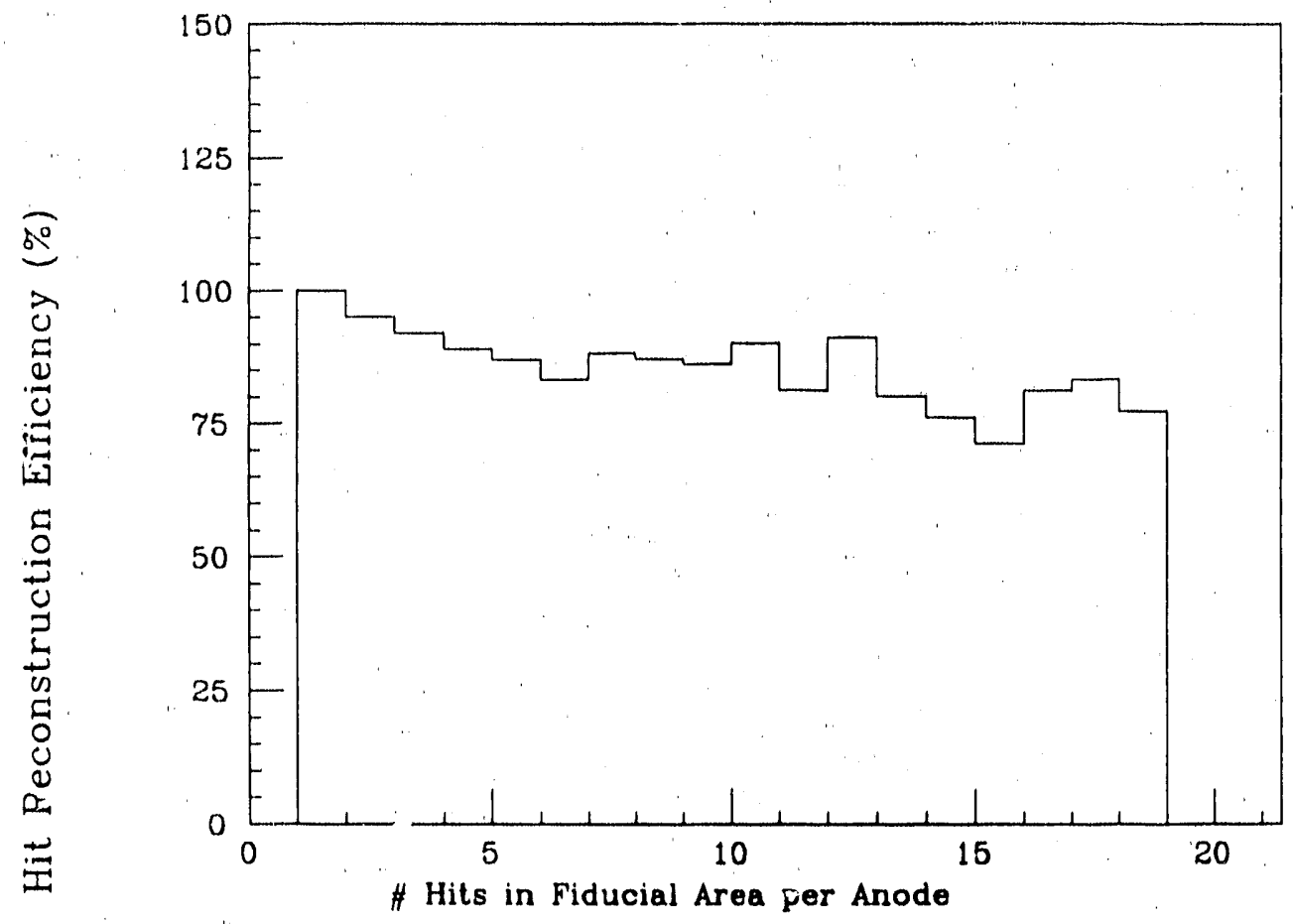

Figure 3-10: The efficiency for identifying and fitting a hit signal in the presence of other pileup hits is shown. Here the method of identifying a hit uses a center of gravity calculation about a peak, and subtraction according to the know amplitude for a minimum ionizing hit.

results shown in Figure $3-10$. In the 1-hit case, this efficiency is understandably $100 \%$, dropping of by approximately $5 \%$ over each of the next few hits, and reaching a plateau at about $75-80 \%$. Considering the very high multiplicities the results are encouraging.

Simply because one is able to fit a hit says nothing of the usefulness of the fit. In Figure 3-11 is shown the sigma in the error of the hit reconstruction, starting out from 1 to 18 hits per anode. Suprisingly, the reconstrustion error plateaus to a value of 10 ns for large numbers of hiss. corresponding to a position error of 80 microns. Clearly. the final reconstruction error one obtains will depend on the reconstruction algorithm used. 


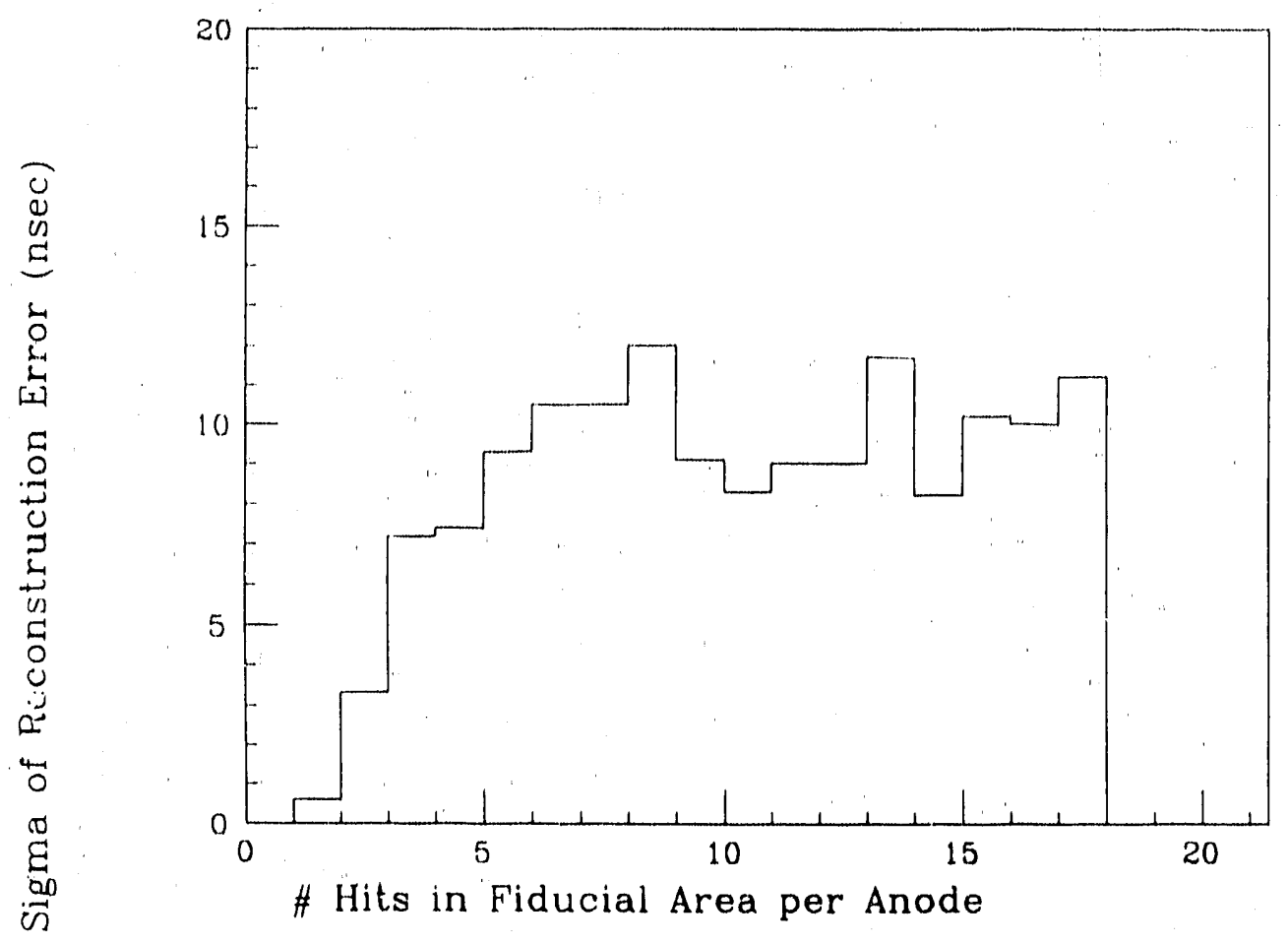

Figure 3-11: The sigma on the accuracy of identifying the drift time when a hit signal is fitted in the presence of other pileup hits is shown. Here the method of identifying a hit uses a center of gravity calculation about a peak, and subtraction according to the know amplitude for a minimum ionizing hit.

\section{Test Program}

The main emphases of our test program will be three-fold:

- 10 finish our tests on the UA6 prototype detector

- 10 begin testing the new cylindrical detector

- to develop and fabricate a prototype detector which can be used for the RHIC OASIS vertex determination (with the help of BNL-Rehak) and then test it

As implied in the last section, a number of tests remain to be done on the protolype detector. These include two-track resolution. iemperature dependence of $\mu_{H^{\prime}}$ B-field and drift-field dependence. and multi-hit response.

These same tests would be carried out on the cylindrical NA45 detector. This detector is a 7 
$\mathrm{cm}$ diameter disk with a small hole (several millimeters in diameter) at the center to allow the primary beam to pass through (it would normally be used in an application where it was concentric about the beam. e.g. as an "endcap" tracker in a central tracking detector). The detector is read out by $36 n$ anodes (one per degree) placed at the outer circumference. In our initial tests, we would use the NA45 readout electronics which is made up of conventional hybrids, which is the same electronics we are presently using for the prototype tests.

In the OASIS experiment at RHIC, we propose to use silicon drift detectors to make a highprecision primary vertex determination to be used in particle tracking. In order to achieve sufficient precision for the vertex position we need position resolution at the drift detectors of 10-20 microns for each coordinate. As we wish to minimize the amount of material between the vertex point and the tracking detectors, the silicon drift detectors are to be placed along the beam axis and recessed from our magnet pole faces. Hits in one of the paired detectors will be matched with those of a second paired detector placed either in front of or behind the first. These matched hits will then point back to the interaction vertex. As the principal investigator and BNL are collaborators on OASIS, we will work together to develop and fabricate detectors at BNL, the testing then being done at both Pittsburgh and BNL using both laser and in-beam tests.

\section{Personnel and Schedule}

The personnel who have been involved with this project and the percentage of their time devoted to it are the following: T. J. Humanic (Principal Investigator, 40\%). M. Clemen (Postdoc, 100\%), D. Kraus (Postdoc, 30\%), and G. Vilkelis (graduate student, 30\%), D. Kraus and G. Vilkelis started on this project in the Summer of 1989. M. Clemen joined the project at the beginning of January. 1990. Clemen will be leaving at the end of 1990 at which point Kraus will become $66 \%$ to $100 \%$ involved with the project. Vilkelis will also continue to be 
involveld. It is my desire to add a full-time graduate student to the project who will get his/hell degree from work mostly done on silicon drift chambers. Such a degree would be at the PH. D. level, in instrumentation, and there are precedents that exist in the Physics Departinent for this.

The sthedule for testing is 10 first finish up the prototype detector and then commence with i.

the lesting of the cylindrical detector. It is assumed that the prototype testing will finish by December, 1990. At this time. the cylindrical detector should become availaible to us. Planning for the OASIS prototype will begin in November of 1990 . 


\section{References}

1. E. Gatti and P. Rehak. Nucl.Instr.and Meth. 225, 608 (1984),

2. P. Rehak et al. Nucl.Instr.and Meth. 248. 367 (1986).

3. E. Gatti et al., Nucl.Instr.and Meth. 274. 469 (1989).

4. C. Jacoboni el al., Solid-State Elect. 20. 77 (1977).

5. D. Long. Phys.Rev. 120. 2024 (1960). 


\section{Budget}

The funding request is $\$ 246.962$ for two years. This will be used to support a posidoc (D. Kraus), a full-time graduate student, 10 buy some lest equipment that is needed (sensitive microammeter. gpib bus hardware), help with the fabrication costs of the new prototype detector, buy more readout electronics, for travel to BNL 10 maintain close contact with P. Rehak, for miscellaneous supplies, for electronic shop work, and for university overhead. The support for T. Humanic and G. Vilkelis is from University and/or NSF money. 
7. Vitae of the Principal investigator

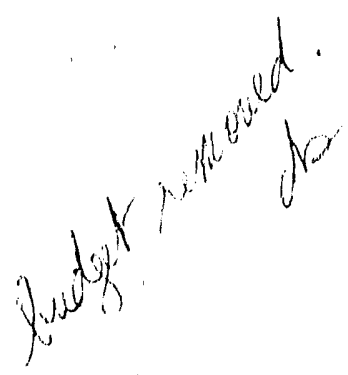



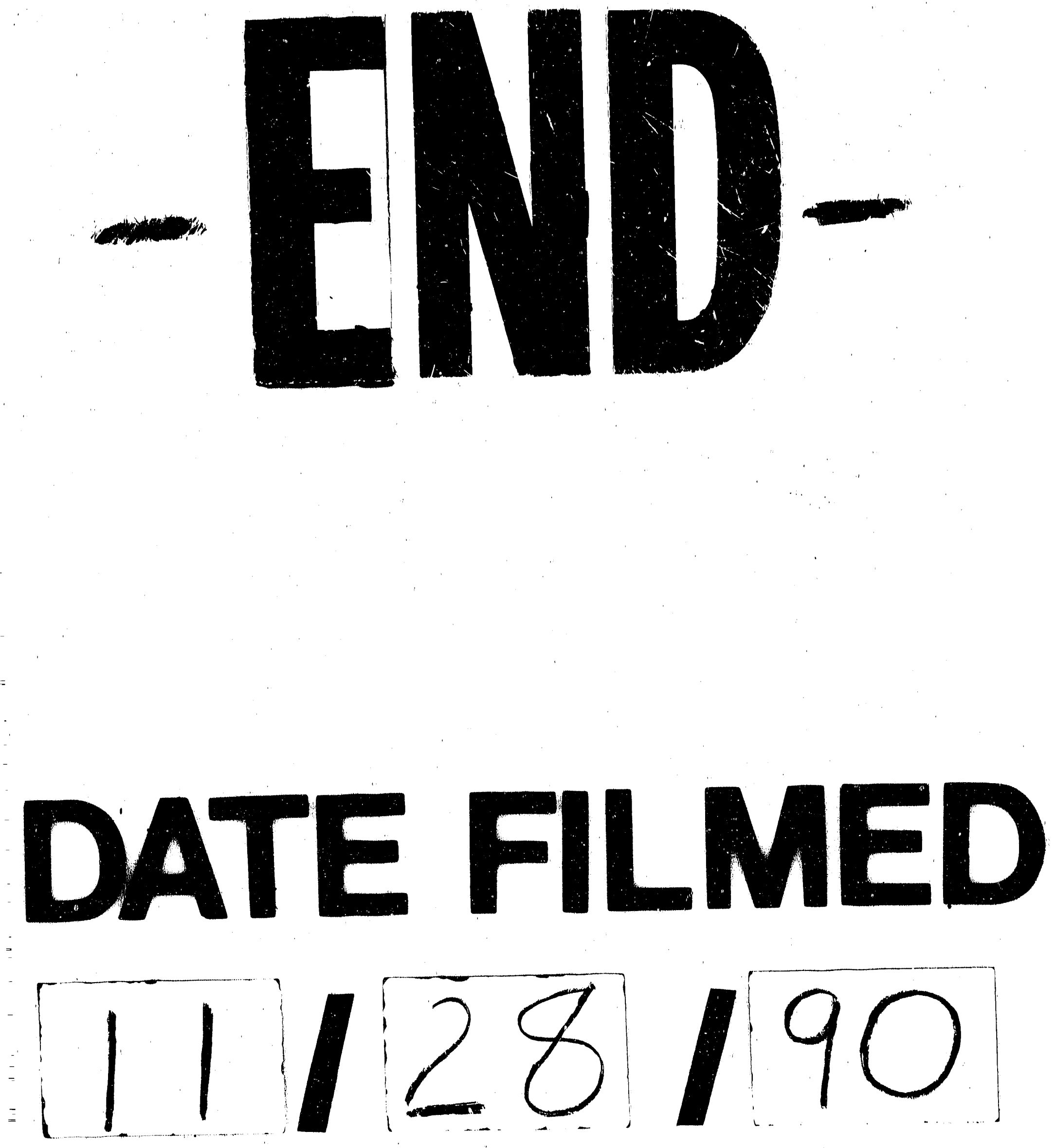